\title{
The Expectancy Dynamics of Anti-Tonal Twelve-Tone Rows: A Commentary and Reanalysis of von Hippel \& Huron (2020)
}

\author{
NIELS CHR. HANSEN[1] \\ Aarhus Institute of Advanced Studies \& Center for Music in the Brain, Aarhus University
}

\begin{abstract}
This commentary provides two methodological expansions of von Hippel and Huron's (2020) empirical report on (anti-)tonality in twelve-tone rows by Arnold Schoenberg, Anton Webern, and Alban Berg. First, motivated by the theoretical importance of equality between all pitch classes in twelve-tone music, a full replication of their findings of "anti-tonality" in rows by Schoenberg and Webern is offered using a revised tonal fit measure which is not biased towards row-initial and row-final subsegments. Second, motivated by a long-standing debate in music cognition research between distributional and sequential/dynamic tonality concepts, information-theoretic measures of entropy and information content are estimated by a computational model and pitted against distributional tonal fit measures. Whereas Schoenberg's rows are characterized by low distributional tonal fit, but do not strongly capitalize on tonal expectancy dynamics, Berg's rows exhibit tonal traits in terms of low unexpectedness, and Webern's rows achieve anti-tonal traits by combining high uncertainty and low unexpectedness through prominent use of the semitone interval. This analysis offers a complementary-and arguably more nuanced-picture of dodecaphonic compositional practice.
\end{abstract}

Submitted 2020 August 21; accepted 2020 August 25.

Published 2020 October 22; https://doi.org/10.18061/emr.v15i1-2.7806

KEYWORDS: dodecaphony, twelve-tone music, tonality, expectation, entropy, probability, information theory

DESPITE Arnold Schoenberg's (1941/1950) proclamation that "[c]omposition with twelve tones has no other aim than comprehensibility" (p. 103), dodecaphonic (i.e., twelve-tone) music has failed to reach the level of widespread popularity that he and his followers from the Second Viennese School had originally hoped for. As we approach the centennial for the discovery of Schoenberg's "method of composing with twelve tones," discussions about its comprehensibility continue to resurface in academic circles. Music cognition researchers, in particular, have contributed to this debate and sometimes provided important empirical corroboration (see, e.g., Brown, 2016; Dibben, 1994; Dienes \& Longuet-Higgins, 2004; de Lannoy, 1972; Krumhansl, Sandell, \& Sergeant, 1987; Ockelford \& Sergeant, 2013; Pedersen, 1975).

The authors of the current target article, Paul von Hippel and David Huron, made an essential contribution to this endeavor when they presented their initial analysis of anti-tonal (or "contra-tonal") traits in Schoenberg's twelve-tone rows in an unpublished conference paper about 20 years ago (Huron \& von Hippel, 2000). Although Huron briefly revisited these results in the penultimate chapter of his Sweet Anticipation (Huron, 2006), the recent paper by von Hippel and Huron (2020) provides the first detailed account in journal publication format and, furthermore, consolidates the original findings by expanding them to Anton Webern's and Alban Berg's rows and by incorporating a set of control variables and a larger control corpus.

A major methodological innovation of this research project is the procedure for quantifying the tonality of twelve-tone rows. Specifically, tonal fit is operationalized as the average of 44 Pearson correlations between the highest-scoring major/minor key profile (see their Figure 2) and sub-segments of cardinality one to eleven - always including the first pitch in the row — of the prime (P), retrograde (R), inversion (I), and retrograde-inversion (RI) forms of each twelve-tone row. In this way, von Hippel and Huron (2020) were able to demonstrate that segments from twelve-tone rows by Arnold Schoenberg $(n=$ $42)$ and Anton Webern $(n=21)$ achieved significantly lower tonal fit than segments from randomly 
generated twelve-tone rows $(n=1,000)$. There was, on the other hand, no significant difference in tonal fit between the random sample and twelve-tone rows by another famous Schoenberg student, Alban Berg $(n=$ 23), who is known for adhering more closely to tonal traits in his dodecaphonic works (Jarman, 1985). These findings were interpreted to mean that Schoenberg's and Webern's twelve-tone rows are in fact "anti-tonal," i.e. actively composed to avoid tonal features and thus presumably also to circumvent tonal listening schemata.

As it provides a methodologically sound empirical basis for discussions about tonality (and absence thereof) in dodecaphonic composition, von Hippel and Huron's (2020) paper was most certainly worth the long wait. At the same time, their choice of method - which is clearly only one amongst many possible ones - invites for replication using alternative procedures. This may challenge or indeed further substantiate their claims regarding anti-tonality in Schoenberg and Webern (and tonality in Berg for that matter). To that end, below, I offer two methodological expansions of von Hippel and Huron's (2020) analysis. First, I present a close replication of their analysis using a modified operationalization of tonal fit that considers all sub-segments of twelve-tone rows on equal terms rather than having a bias towards initial and final tones of the rows. Second, I quantify the sequential expectancy dynamics of twelve-tone row forms using a computational model of melodic expectation. As will be evident, some of these analyses are consistent with von Hippel and Huron's (2020) findings while others deviate slightly and thus may offer a more nuanced picture of anti-tonal and tonal twelve-tone composition in Schoenberg's, Webern's, and Berg's oeuvres.

\section{CONSIDERING ALL POSSIBLE SUB-SEGMENTS OF ROWS}

As mentioned above, von Hippel and Huron's (2020) operationalization of tonal fit averages 44 correlations corresponding to the highest numerical Pearson correlation with any of the 12 major or 12 minor key profiles (cf. Krumhansl \& Kessler, 1982) for the 1 to 11 first tones of the P, I, R, and RI forms of each row. Because peripheral (i.e., initial or final) tones of the row are more likely than medial tones to be featured in sub-segments computed in this way, this automatically leads to an imbalance in favor of the former over the latter. This resulting row-initial and row-final bias may indeed be justified in terms of general psychological mechanisms where primacy and recency effects are well-established (Lewandowsky \& Murdock, 1989) and in musical practice where themes are often identified by their beginning, as exemplified by dictionaries of musical themes (e.g., Barlow \& Morgenstern, 1983). Musical serial order effects also appear, for example, in the reconstruction of order for verses in church hymns (Maylor, 2002). Yet, the current operationalization of tonal fit seems somewhat at odds with dodecaphonic principles of equality between all twelve pitch classes, such as expressed in Josef Matthias Hauer's (1920) "law of the twelve tones" which inspired subsequent dodecaphonic compositional practice. Thus, it seems relevant to test if von Hippel and Huron's (2020) findings hold true with an alternative operationalization of tonal fit that considers all possible sub-segments of the rows.

\section{Methods}

All the twelve-tone rows used in the original study by von Hippel and Huron (2020) were obtained in the humdrum **kern format. Specifically, 42, 21, and 23 rows composed by Arnold Schoenberg, Anton Webern, and Alban Berg, respectively, were downloaded from the website of the Center for Computer Assisted Research in the Humanities (London et al., 2002). The authors of the target article, moreover, provided a link to the 1,000 random rows used in their study.

All available files were imported into R (R Core Team, 2018) and processed there. First, a **kern spine was added to the random row files who previously only contained a **pc spine with numerals $0-11$ to designate 12-tet pitch classes. The **kern spine was created by (arbitrarily) mapping 0 to $\mathrm{C}, 1$ to $\mathrm{C \# ,} 2$ to $\mathrm{D}$ etc. Second, an arbitrary quarter-note duration was added to all note events (this was required for import into the computational model used below). Third, retrograde (R), inversion (I), and retrograde inversion (RI) forms of each row were generated from the imported prime (P) forms. Specifically, the order of pitches of the $\mathrm{P}$ form was reversed to obtain the $\mathrm{R}$ form. To obtain the I form, the intervals of the $\mathrm{P}$ form were first inverted after which the new row was transposed up or down in its entirety to comprise the exact same pitch set as the $\mathrm{P}$ form (i.e., all pitches from C3 to B3). For consistency, the **pc spine was updated 
accordingly. Finally, the RI form was obtained by reversing the order of pitches in this newly obtained I form.

Next, for replication purposes, von Hippel and Huron's (2020) original measure of tonal fit was computed. This was done by extracting the 11 sub-segments of cardinality 1 to 11 (i.e., comprising the first tone, the first two tones, the first three tones etc.) of each of the four P, R, I, RI forms of each twelve-tone row. The Krumhansl-Schmuckler key-finding algorithm (Krumhansl \& Schmuckler, 1986; Krumhansl, 1990) was then applied to each of these 44 sub-segments to identify the highest scoring Pearson correlation with any of the 12 major and 12 minor key profiles (Krumhansl \& Kessler, 1982). Tonal fit was finally calculated by averaging these 44 values.

Finally, an alternative operationalization of tonal fit was computed that equally considers all possible sub-segments of rows, thus avoiding the bias for peripheral over medial tones in von Hippel and Huron's (2020) original measure. This novel tonal fit measure was based on a total of 130 (rather than 44) Pearson correlation values for each row, corresponding to all the possible 11, 10, 9, 8, 7, 6, 5, 4, 3, and 2 sub-segments of consecutive tones of cardinalities 2 to 11, respectively, for the P and I forms. R and RI forms were not used as sub-segments would be identical between the P and R as well as between the I and RI forms. Cardinalities 1 and 12 were also excluded as the resulting correlation values of single pitches or full chromatic sets with the key profiles would merely have changed the tonal fit measure by a constant.[2] Apart from the initial selection of a larger number of sub-segments, the alternative operationalization of tonal fit was calculated similarly to the previous measure by averaging the 130 highest-scoring Pearson correlations with the Krumhansl-Kessler key profiles.

\section{Results}

Figure 1 contains violin plots of the replication of von Hippel and Huron's (2020) results with their original tonal fit measure (left panel) as well as the results from the new analysis with the novel tonal fit measure introduced above (right panel). Unlike the analysis reported by von Hippel and Huron, the measures reported here were not standardized to the mean and standard deviation of random rows but rather occupy the original scale typical of correlation values (i.e., theoretically ranging from -1 to 1 although the selection of the highest correlations makes negative tonal fit values extremely unlikely).

To assess if the twelve-tone rows by the three specific composers differed significantly from random rows, linear regression with robust (i.e., heteroscedasticity-resistant) standard errors was conducted predicting the original and revised tonal fit measures with dummy variables for each of the composers using the $\operatorname{lm}($ ) function in R (R Core Team, 2018).[3] Similar to the previous study, quantile regression was also conducted to compare the median between the different types of rows. To this end, the $r q()$ function from the quantreg package was used (Koenker, 2020).

As evident from Tables 1 and 2, both types of regression confirmed that Alban Berg's twelve-tone rows did not differ significantly from the random rows neither when tonal fit was calculated as done by von Hippel and Huron (2020) nor when using the revised measure introduced here. The rows by Arnold Schoenberg and Anton Webern, however, showed significantly lower levels of tonal fit than the random rows, and this was the case for both the original and revised measures with both types of regression. Taken together, these follow-up analyses indicate that the effects for anti-tonality in dodecaphonic compositions by Schoenberg and Webern hold true when all row members are considered equally. 

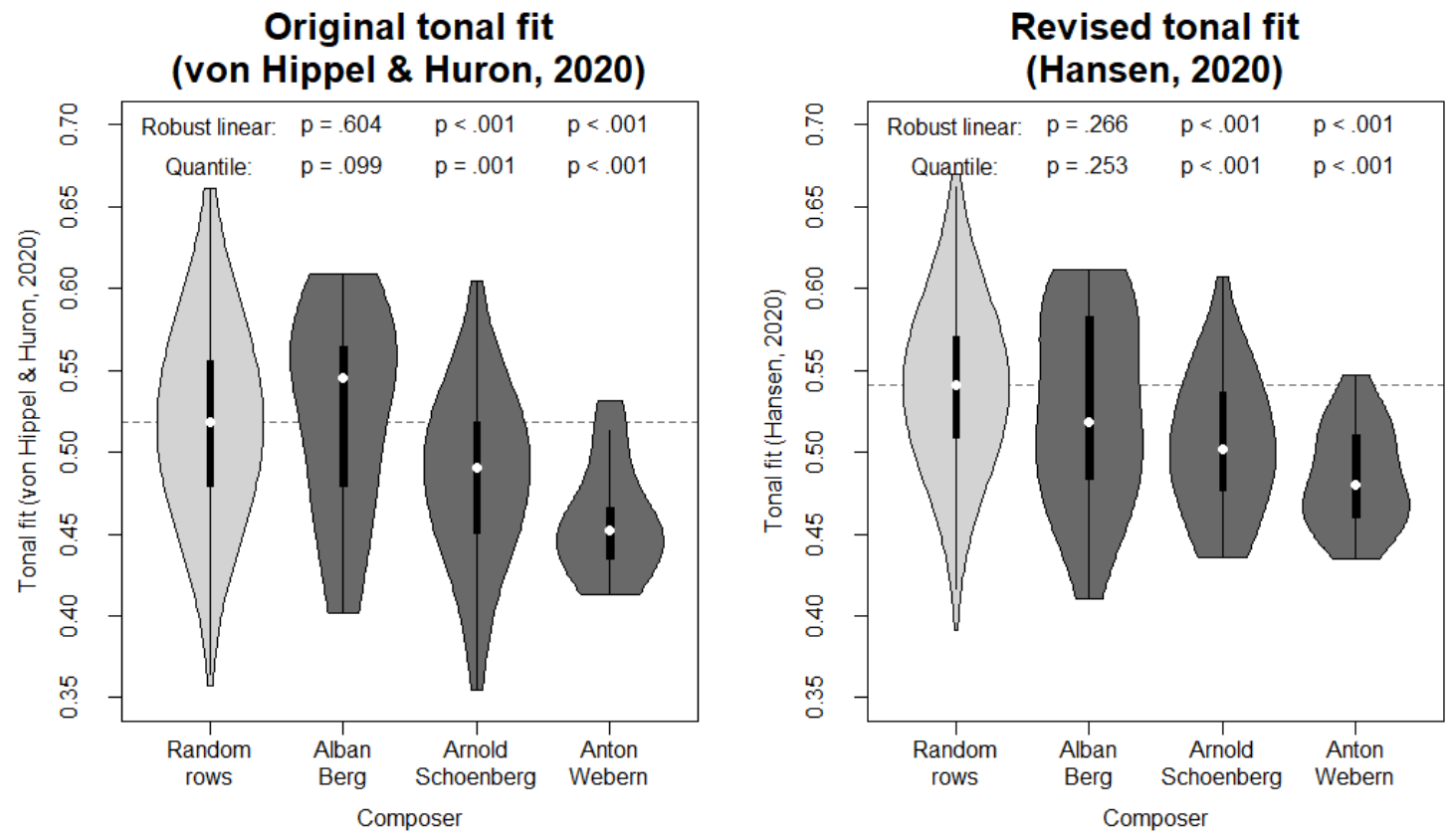

Figure 1. Violin plots of the tonal fit values for the 1,000 random twelve-tone rows as well as the 23, 42, and 21 twelve-tone rows composed by Alban Berg, Arnold Schoenberg, and Anton Webern, respectively. The violins comprise a kernel density plot displayed on top of a conventional boxplot with median (white dot), interquartile range (black box), and lower/upper adjacent values (whiskers). The left panel depicts von Hippel and Huron's (2020) original tonal fit measure whereas the right panel depicts the revised tonal fit measure introduced here which is not biased towards row-initial and row-final sub-segments. As can be seen, the revised measure replicates the original findings that, in comparison with random rows, Schoenberg's and Webern's rows were significantly anti-tonal whereas Berg's rows were not.

Table 1. Linear regression with robust standard errors (left) and quantile median regression (right) of the original tonal fit measure by von Hippel and Huron (2020).

Linear robust regression

\begin{tabular}{lrrr} 
& Coefficient (SE) & $t$ & $p$ \\
\hline Intercept & $0.52(<0.01)$ & 310.24 & $<.01$ \\
Berg & $0.01(0.01)$ & 0.52 & .60 \\
Schoenberg & $-0.03(<0.01)$ & -4.12 & $<.01$ \\
Webern & $-0.06(<0.01)$ & -8.09 & $<.01$ \\
\hline
\end{tabular}

Quantile (median) regression

\begin{tabular}{rrr} 
Coefficient $(\mathrm{SE})$ & $t$ & $p$ \\
\hline $0.52(<0.01)$ & 220.03 & $<.01$ \\
$0.03(0.02)$ & 1.65 & .10 \\
$-0.03(<0.01)$ & -3.40 & $<.01$ \\
$-0.07(<0.01)$ & -7.63 & $<.01$ \\
\hline
\end{tabular}

Table 2. Linear regression with robust standard errors (left) and quantile median regression (right) of the revised tonal fit measure introduced here.

\begin{tabular}{lrrrrrrrr} 
& \multicolumn{3}{c}{ Linear robust regression } & & \multicolumn{2}{c}{ Quantile (median) regression } \\
& Coefficient $(\mathrm{SE})$ & $t$ & $p$ & & Coefficient (SE) & $t$ & $p$ \\
\hline Intercept & $0.54(<0.01)$ & 384.98 & $<.01$ & & $0.54(<0.01)$ & 308.13 & $<.01$ \\
Berg & $-0.01(0.01)$ & -1.11 & .27 & & $-0.02(0.02)$ & -1.14 & .25 \\
Schoenberg & $-0.04(<0.01)$ & -5.44 & $<.01$ & & $-0.04(<0.01)$ & -3.96 & $<.01$ \\
Webern & $-0.06(<0.01)$ & -8.42 & $<.01$ & & $-0.06(<0.01)$ & -6.60 & $<.01$ \\
\hline
\end{tabular}




\section{CONSIDERING SEQUENTIAL EXPECTANCY DYNAMICS IN TONALLY ENCULTURATED LISTENERS}

Following in the footsteps of an old discussion in music cognition research regarding whether to operationalize tonality in distributional (e.g., Krumhansl, 1990) or sequential terms (e.g., Butler, 1989), von Hippel and Huron's (2020) method can rightfully be criticized for adhering to the former rather than the latter approach.[4] Neither the original tonal fit measure nor the revised one evaluated above considers the order in which row members occur. Yet, as music unfolds in time, and the human brain engages in "eager processing" whereby incoming sensory input is continually recoded as soon as it arrives (Christiansen \& Chater, 2016), perception of musical structure is necessarily sequential rather than retrospectively summative (Hansen, Kragness, Vuust, Trainor, \& Pearce, under review).

Specifically in the context of dodecaphonic composition, it has previously been argued that there is a cognitive dimension to understanding the aesthetics and reception of twelve-tone music as well as the historical context in which it emerged. An unbalanced focus on compositional process over perceptual structure - what Taruskin (2004) refers to as "the poietic fallacy"- has undoubtedly not benefitted the popularity of twelve-tone music in the general public. In his discussion of cognitive constraints on compositional systems, Lerdahl (1992) argued that the serial organization of musical sounds as found in many twelve-tone pieces made this music "cognitively opaque" from a listener's perspective. This opaqueness was primarily ascribed to serialism's permutational rather than elaborational melodic structure, its avoidance of sensory consonance and dissonance distinctions, and its inability to induce a pitch space mapping spatial distance to cognitive distance. Along similar lines, other accounts have emphasized how key features of dodecaphonic composition like flat zeroth-order pitch distributions, registral discontinuity (resulting from octave equivalence), limited hierarchical depth of motives, weak metrical scaffolding, rhythmical irregularity, and the lack of well-established cadential formulas and repetitions transgress fundamental principles of music listening (Ball, 2011; Lerdahl, 2001). Humans are, in other words, predisposed for musics that lend themselves well to our cognitive chunking mechanisms, limited working memory capacity, preference for familiar structures, and sophisticated statistical learning and pattern discovery skills.

A growing body of research indeed demonstrates that expectations play a key role in all of these areas. This is, for example, the case for segmentation of music into phrases (Pearce, Müllensiefen, \& Wiggins, 2010; Hansen, Kragness, Vuust, Trainor, \& Pearce, under review), memory for melodies (Agres, Abdallah, \& Pearce, 2018), stylistic enculturation (Hansen, Vuust, \& Pearce, 2016; Morrison, Demorest, \& Pearce, 2018), and the cognition of musical repetition (Margulis, 2014) and cadence formulas (Sears, Pearce, Caplin, \& McAdams, 2018; Sears, Pearce, Spitzer, Caplin, \& McAdams, 2019). Thus, claims about anti-tonality in dodecaphonic composition ultimately makes assumptions about psychological expectation mechanisms rather than about musical structure per se (i.e., independent from the listener's perspective). While the key profiles used for computing tonal fit measures above were indeed empirically derived from listening experiments with human participants, they were not used in a way where the order of pitches in the melodic sequence was considered, and they only assessed listener expectations at one particular point in a listening episode. The expectancy dynamics of twelve-tone rows can be modelled more dynamically with computational models of event-based listening such as the one adopted below.

\section{Methods}

The Information Dynamics of Music Model (IDyOM, Pearce, 2005) was used to estimate event-level measures of information content (IC) and entropy for the P, R, I, and RI forms of the 1,000 random and 86 composed twelve-tone rows. The information-theoretic estimates of information content and entropy from this unsupervised, variable-order $n$-gram model have previously been shown to relate directly to the unexpectedness and uncertainty experienced by human listeners (Hansen \& Pearce, 2014). For the main analysis, short-term models making predictions from the local context (i.e., the relevant form of the twelvetone row in question) were combined with long term-models trained on a large corpus of European folksongs. This sizeable corpus of 279,926 notes from a total of 5,739 melodies taken from the fink, erk, boehme, ballad, allerkbd, altdeu, dva, zuccal, and kinder subsets of German folksongs and the further subsets of Alsatian, Yugoslavian, Swiss, and Austrian folksongs from the Essen Folksong Collection 
(Schaffrath, 1992, 1993) ensured that the resulting information-theoretic measures were representative of tonal listening schemata at large.

IDyOM was configured to predict the absolute pitch of each event in the sequence using the cpint viewpoint. This viewpoint represents each event as a single numeric indicating the relative pitch interval in semitones from the pitch of the preceding note in the sequence. In previous work, this viewpoint has often been combined with the cpintfref viewpoint representing the chromatic scale-degree of a given note in relation to the overall tonic and mode of the musical piece in question (e.g., Hansen \& Pearce, 2014; Hansen, Vuust, \& Pearce, 2016). As scale-degrees cannot, however, be defined meaningfully for twelve-tone rows, the current analysis resorted to the remaining, well-defined cpint viewpoint.

Before conducting robust linear regression and median regression of the information-theoretic measures as presented above for the original and revised tonal fit measures, mean entropy and IC values were computed for each of the random and composed rows. This was done by averaging across all noninitial events in any of the four P, R, I, and RI forms. Entropy and IC for initial note events in each form of each row were disregarded as the lack of a prior context means that IDyOM, in these cases, estimates from a uniform probability distribution resulting in constant row-initial entropy and IC levels.

\section{Results}

As an initial sanity check, the overall success of each execution of IDyOM (configured as described above) in predicting the random and composed twelve-tone rows was compared with the success of IDyOM executions where the long-term models were trained on the row corpora themselves (via 10-fold crossvalidation) rather than on a large, tonal corpus as described above. These alternative models can be viewed as simulating the experience of an expert listener skilled in (only) twelve-tone rows. To this end, model success was quantified in terms of average information content where low values signify that the notes that actually occurred in the sequence were predicted by the model with high probability on average whereas high values, conversely, signify that the model was relatively surprised about the notes in the sequence.

Unsurprisingly, this analysis found that random and composed rows were both predicted more strongly by IDyOM models trained on the rows themselves (mean $\mathrm{IC}_{\text {random: }}$ : 3.49 ; mean $\mathrm{IC}_{\text {composed }}$ : 3.38 ) than by IDyOM models trained on the (stylistically somewhat irrelevant) tonal corpus (mean $\mathrm{IC}_{\text {random: }}$ : 5.82; mean $\mathrm{IC}_{\text {composed: }}$ 5.60). The fact that mean IC was lower for composed than for random rows both when predicted by dodecaphonic IDyOM models and by tonal IDyOM models suggests that composed rows contained more systematic combinations of pitch intervals than random rows - both in the context of twelve-tone music and tonal folksongs. While consistent with von Hippel and Huron's (2020) suggestion that Alban Berg may have composed rows with tonal characteristics, these preliminary findings do not overall seem consistent with their conclusions about anti-tonality in Schoenberg's and Webern's music. To assess these issues in greater detail, however, one must proceed to distinguishing between the rows by each of the three twelve-tone composers.

As for the tonal fit measures analyzed above, linear regression with robust standard errors as well as quantile (median) regression were conducted for the two information-theoretic measures IC and entropy (Figure 2 and Tables 3 and 4). This analysis found that to a listener enculturated in tonal music, Alban Berg's and Anton Webern's twelve-tone rows generally contained more expected notes than was the case for the random rows. Arnold Schoenberg's rows, on the other hand, did not show significantly different levels of IC in comparison with the random control corpus. Moreover, while Berg's and Schoenberg's twelve-tone rows were no different from the random rows in terms of mean entropy, Webern's rows were generally estimated to evoke more uncertain pitch expectations in tonal listeners. It will be discussed below how these results differ in interesting ways from von Hippel and Huron's (2020) findings without being incompatible with them. 
Unexpectedness

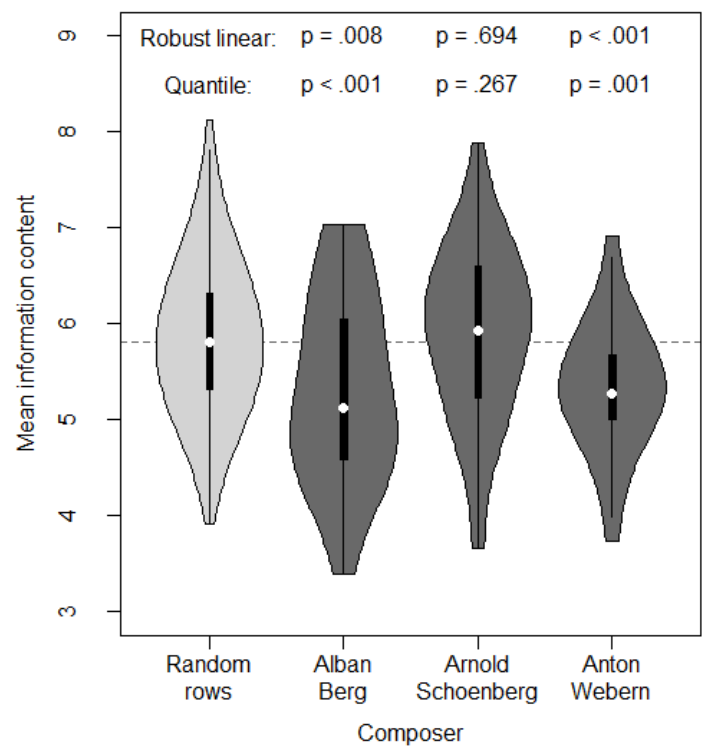

\section{Uncertainty}

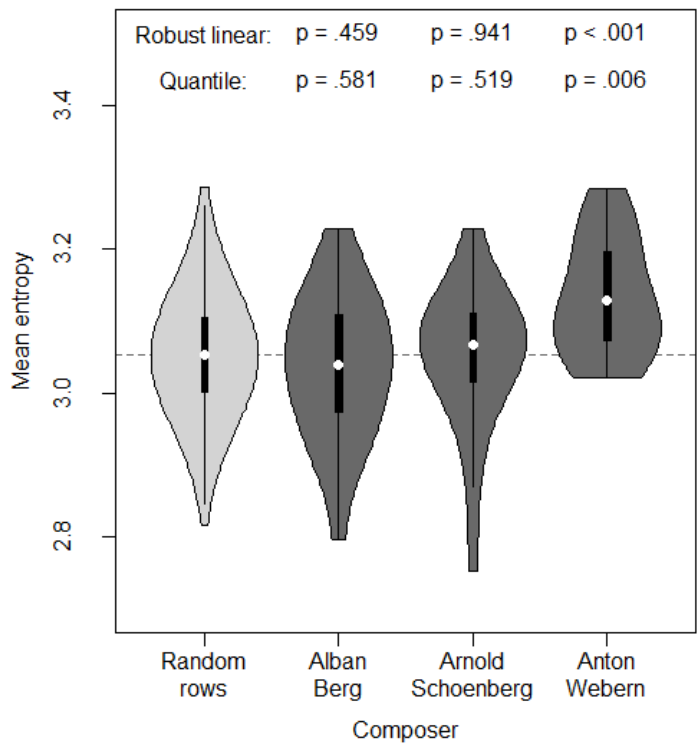

Figure 2. Violin plots of the information-theoretic measures estimated by IDyOM for the 1,000 random twelve-tone rows as well as the 23, 42, and 21 twelve-tone rows composed by Alban Berg, Arnold Schoenberg, and Anton Webern, respectively. The violins comprise a kernel density plot displayed on top of a conventional boxplot with median (white dot), interquartile range (black box), and lower/upper adjacent values (whiskers). The left panel depicts the mean information content which quantifies the average unexpectedness of notes in the row whereas the right panel depicts the mean entropy which quantifies the average uncertainty when listeners make predictions about the next pitch in the row. As can be seen, for listeners accustomed to tonal music, both Alban Berg's and Anton Webern's twelve-tone rows were significantly more expected than random rows whereas Anton Webern's rows simultaneously created more uncertain expectations in listeners. Arnold Schoenberg's rows did, on the other hand, not differ from random rows in terms of these information-theoretic measures.

Table 3. Linear regression with robust standard errors (left) and quantile median regression (right) of the information content measure estimated by IDyOM.

Linear robust regression

\begin{tabular}{lrrr} 
& Coefficient (SE) & $t$ & $p$ \\
\hline Intercept & $5.85(0.02)$ & 258.11 & $<.01$ \\
Berg & $-0.53(0.20)$ & -2.64 & $<.01$ \\
Schoenberg & $0.05(0.14)$ & 0.39 & .69 \\
Webern & $-0.51(0.15)$ & -3.43 & $<.01$ \\
\hline
\end{tabular}

Quantile (median) regression

\begin{tabular}{rrr} 
Coefficient (SE) & $t$ & $p$ \\
\hline $5.82(0.03)$ & 169.95 & $<.01$ \\
$-0.69(0.19)$ & -3.65 & $<.01$ \\
$0.12(0.11)$ & 1.11 & .27 \\
$-0.54(0.17)$ & -3.19 & $<.01$ \\
\hline
\end{tabular}

Table 4. Linear regression with robust standard errors (left) and quantile median regression (right) of the entropy measure estimated by IDyOM.

Linear robust regression

\begin{tabular}{lrrr} 
& Coefficient (SE) & $t$ & $p$ \\
\hline Intercept & $3.05(<0.01)$ & 1245.96 & $<.01$ \\
Berg & $-0.02(0.02)$ & -0.74 & .46 \\
Schoenberg & $<0.00(0.02)$ & -0.08 & .94 \\
Webern & $0.08(0.02)$ & 4.52 & $<.01$ \\
\hline
\end{tabular}

Quantile (median) regression

\begin{tabular}{rrr} 
Coefficient (SE) & $t$ & $p$ \\
\hline $3.05(<0.01)$ & 1009.75 & $<.01$
\end{tabular}

$\begin{array}{lll}-0.01(0.02) & -0.55 & .58\end{array}$

$\begin{array}{lll}0.01(0.02) & 0.64 & .52\end{array}$

\begin{tabular}{lll}
$0.08(0.03)$ & 2.75 & $<.01$ \\
\hline
\end{tabular}


Additionally, to evaluate the relative contribution of tonal fit, expectancy dynamics, and control variables representing other structural row properties, multiple logistic regression analysis was conducted, following von Hippel and Huron's (2020) procedure of adding all relevant predictors at once (i.e., the "enter" method). All the original control variables were added as predictors, including binary variables for whether rows were (a) inversionally hexachordally combinatorial (IHC), (b) derived from trichords, tetrachords, pentachords, or hexachords, and/or (c) mirror-symmetric, as well as numeric variables for how many (d) semitones and trichords of the (e) (014) and (f) (016) type they contained. In contrast to the target authors' analysis, the revised (unstandardized) tonal fit measure introduced above replaced the original one, and mean information content (IC) and mean entropy were also added to model expectancy dynamics.

The results from the logistic regression analysis complements von Hippel and Huron's findings in a number of interesting ways (Table 5). First, the Schoenberg analysis replicates the findings reported in the original paper and expanded upon here, namely that his rows exhibit significantly lower tonal fit than random, but that mean IC and entropy do not contribute significantly to predicting their status as composed. Moreover, the previously significant tendency for more semitone intervals did no longer meet the significance threshold, thus indicating that some of this variance may have been accounted for by the added (albeit non-significant) information-theoretic predictors. The tendencies for lower entropy and higher chromaticism in Schoenberg's rows both achieved marginally non-significant $p$-values of .13 and .11 , respectively.

Second, similar to what von Hippel and Huron reported, Webern's rows exhibited significantly lower degrees of tonal fit and significantly more semitone intervals and (014) trichords than the random rows. Although entropy was higher and IC was lower in Webern's rows, the related variance could better be explained by these other variables. To explore the patterns of overlapping variance explained, individual logistic regression models were fitted with mean IC and mean entropy in combination with only one of the measures revised tonal fit $(A I C=147.99)$, $(014)$ trichords $(A I C=152.71)$, or semitones $(A I C=136.40)$. Whereas mean IC and entropy remained significant predictors when combined with tonal fit and (014) trichords (all. $p$ 's $<.03$ ), their contribution became highly non-significant when combined with number of semitones as predictor (both $p$ 's $>.92$ ). The fact that semitones and the information-theoretic measures explained overlapping variance was additionally supported by the fact that a model with number of semitones as the only predictor achieved lower AIC than any of the other models $(A I C=132.42)$.

Third and finally, the only significant predictor for Berg's rows was mean IC which was significantly lower than for random rows. The revised tonal fit measure, on the other hand, did not achieve statistical significance. Interestingly, thus, while this could not be established with the distributional tonal fit measures, the lower average surprise evoked by note transitions in the Berg corpus is indeed consistent with the widespread consensus that Berg's twelve-tone rows tend to show tonal characteristics (Headlam, 1996). The fact that mirror symmetry and semitone predictors were no longer significant after the addition of mean IC suggests that low pitch unexpectedness was more predictive of Berg's rows than semitone intervals and symmetrical structure per se. 
Table 5. Multiple logistic regression analysis predicting whether rows were random or composed with the revised tonal fit measure, the information-theoretic measures, and the control variables entered as predictors (using the "enter" method), separately for each of the three composers.

\begin{tabular}{|c|c|c|c|c|c|c|}
\hline \multirow[b]{2}{*}{ Predictors } & \multicolumn{2}{|c|}{ Schoenberg $(n=42)$} & \multicolumn{2}{|c|}{ Webern $(n=21)$} & \multicolumn{2}{|c|}{$\operatorname{Berg}(n=23)$} \\
\hline & $\begin{array}{r}\text { Odds ratio } \\
{[95 \% \mathrm{CI}]}\end{array}$ & $p$ & $\begin{array}{r}\text { Odds ratio } \\
{[95 \% \mathrm{CI}]}\end{array}$ & $p$ & $\begin{array}{l}\text { Odds ratio } \\
{[95 \% \mathrm{CI}]}\end{array}$ & $p$ \\
\hline \multirow[t]{2}{*}{ Mean IC } & 1.04 & .87 & 0.72 & .51 & 0.38 & $<.01 * *$ \\
\hline & {$[0.64,1.71]$} & & {$[0.28,1.90]$} & & {$[0.19,0.78]$} & \\
\hline \multirow{2}{*}{ Mean entropy } & 0.02 & .13 & 5.93 & .70 & 0.04 & .27 \\
\hline & {$[0,3.15]$} & & {$[0,57904.05]$} & & {$[0,12.94]$} & \\
\hline \multirow[t]{2}{*}{ Tonal fit (rev.) } & $<0.01$ & $<.01 * *$ & $<0.01$ & $.04 *$ & $<0.01$ & .19 \\
\hline & {$[0,0.02]$} & & {$[0,0.28]$} & & {$[0,44.69]$} & \\
\hline \multirow[t]{2}{*}{$\mathrm{IHC}$} & 7.56 & $<.01 * *$ & 0.90 & .87 & 0.84 & .70 \\
\hline & {$[3.05,18.75]$} & & {$[0.26,3.10]$} & & {$[0.34,2.04]$} & \\
\hline \multirow[t]{2}{*}{ Derived } & 2.49 & .33 & 10.10 & $.06 \dagger$ & 3.50 & .22 \\
\hline & {$[0.39,15.79]$} & & {$[0.88,115.76]$} & & {$[0.48,25.58]$} & \\
\hline \multirow[t]{2}{*}{ Mirror symmetric } & 3.21 & .28 & 1.34 & .86 & 7.41 & $.06 \dagger$ \\
\hline & {$[0.38,26.81]$} & & {$[0.06,31.23]$} & & {$[0.95,57.91]$} & \\
\hline \multirow[t]{2}{*}{ \#semitones } & 1.28 & .11 & 2.06 & $<.01 * *$ & 1.23 & .33 \\
\hline & {$[0.94,1.74]$} & & {$[1.21,3.50]$} & & {$[0.81,1.85]$} & \\
\hline \multirow[t]{2}{*}{ \# (014) trichords } & 1.89 & $<.01 * *$ & 3.24 & $<.01 * *$ & 0.49 & .11 \\
\hline & {$[1.27,2.83]$} & & {$[1.68,6.26]$} & & {$[0.21,1.16]$} & \\
\hline \multirow[t]{2}{*}{ \# (016) trichords } & 1.33 & .26 & 1.02 & .97 & 0.92 & .83 \\
\hline & {$[0.81,2.20]$} & & {$[0.43,2.43]$} & & {$[0.44,1.92]$} & \\
\hline Constant & 269078.20 & .11 & 1.28 & .99 & 6935713.99 & $.08 \dagger$ \\
\hline
\end{tabular}

\section{CONCLUSIONS}

Motivated by the theoretical importance of equality between all pitch classes in twelve-tone music and by a long-standing debate in music cognition research between distributional and more dynamic tonality concepts, this commentary provides two methodological expansions of von Hippel and Huron's (2020) empirical report on tonality and anti-tonality in twelve-tone rows by Arnold Schoenberg, Anton Webern, and Alban Berg.

First, a full replication with a novel tonal fit measure without the original bias towards row-initial and row-final sub-segments was presented. The only small deviations from findings with the original measure was that the median revised tonal fit for Alban Berg was no longer numerically higher than that for random rows, and that the original non-significant tendency towards higher-than-random tonal fit for Berg when conducting quantile regression was also no longer present. This suggests that Berg's twelvetone rows are not truly more tonal than random rows when tonality is assessed in distributional terms. Alternatively, tonal traits could be more prominent at the beginning (or end) of the rows, but this speculation would require more detailed testing to be properly established.

Second, the unsupervised, variable-order $n$-gram model IDyOM was trained on a large corpus of tonal folksongs to emulate the expectations of Western-enculturated listeners. Before interpreting the results from these simulations, however, an important methodological caveat remains: the unsurprising finding that the tonally-trained model performed worse than a model trained on the twelve-tone rows themselves raises the question whether listeners truly resort to tonal listening schemata when listening to twelve-tone music. Previous evidence already suggests that listeners may not always revert to a stylistically inappropriate schema even though it would result in stronger predictions than a more stylistically appropriate schema that they have limited training in. Hansen, Vuust, and Pearce (2016), specifically, found that professional classical musicians and non-musicians did not seem to misapply general tonal expectations when listening to bebop solos by Charlie Parker. This finding can be explained in terms of "cognitive firewalls" which restrict the use of internalized probabilistic knowledge by contextual relevance (Cosmides \& Tooby, 2000; Huron, 2006). Future research uncovering the specifics of schematic listening 
behavior for dodecaphonic music may provide important context for the interpretation of the findings presented here.

Keeping the aforementioned methodological caveat in mind, statistical analysis of the IDyOM estimates did indeed seem to provide empirical evidence for von Hippel and Huron's (2020) assertion of "tonally evocative rows" (p. 110) in Alban Berg's dodecaphonic works. Berg's rows contained pitches that were on average more expected than pitches in the corpus of random rows. At the same time, the expectations evoked by Berg's rows were neither more nor less uncertain than those evoked by random rows. Logistic regression with structural control variables, furthermore, confirmed that the higher degrees of expectedness explained variance more effectively than structural factors. This variance was previously accounted for by predictors representing the prominence of semitones and mirror symmetry properties.

Interestingly, Webern's rows generated significantly more uncertain expectations in listeners, but resolved such high degrees of uncertainty with relatively highly expected pitch transitions on average. Because semitone intervals are extremely common in tonal music, this would be consistent with von Hippel and Huron's (2020) finding that Webern's well-known predilection for the semitone interval (Hanson, 1983) extended into his dodecaphonic period. The multiple logistic regression analysis demonstrated very clearly that the number of semitone intervals present in Webern's rows explains nearly the exact same variance as that explained by the combination of high uncertainty and low unexpectedness. Tonal fit, conversely, did explain unique variance not accounted for by the semitone predictor. Thus, it seems that Webern composed twelve-tone rows with relatively many semitone intervals which - as is the case for the leading-tone-to-tonic resolutions in tonal music - are both highly expected and lead to relatively uncertain expectations about what follows next (Huron, 2006). Butler's (1989) characterization of semitones as rare intervals carrying "critical information about local harmonic goals" (p. 233), in other words, manifests in Webern's dodecaphonic pieces in terms of row segments that score high on the distributional tonal fit measures.

Finally, whereas Schoenberg's rows achieved lower distributional tonal fit values and had a greater prominence of (014) trichords and inversional hexachordal combinatoriality than random rows, they did not seem to capitalize on different levels of entropy and information content. Thus, the informationtheoretic analysis overall failed to generalize von Hippel and Huron's findings of distributional anti-tonality in Schoenberg's music to the ongoing expectancy dynamics of tonally-enculturated listeners. This inconsistency perfectly exemplifies how distributional and sequential/dynamic measures are capturing different aspects of tonality that are not necessarily mutually dependent and overlapping.

In his recent review of the long-standing debate about the distributional vs. sequential nature of musical tonality, Schmuckler (2016) concluded that "distributional and structural-functional approaches are really part of the same process, and that a complete description of tonality and key-finding will require both global distributional properties, along with more localized, structural-functional information" (p. 148). The two methodological expansions of von Hippel and Huron's (2020) study offered here contribute fruitfully to both of these analytical perspectives.

\section{ACKNOWLEDGEMENTS}

Thanks to Paul von Hippel and David Huron for providing full access to their materials and for answering clarifying questions about their methodology. This article was layout edited by Kelly Jakubowski.

\section{NOTES}

[1] Correspondence can be addressed to: Dr. Niels Chr. Hansen, Aarhus Institute of Advanced Studies \& Center for Music in the Brain, Aarhus University, Høegh-Guldbergs Gade 6B, 8000 Aarhus C, Denmark, nchansen@aias.au.dk.

[2] The same was the case for von Hippel and Huron's (2020) original tonal fit measure, but these authors still decided to include sub-segments with cardinality 1 in their 44 correlation values. As indicated, this methodological decision had no detrimental effects on the outcome of the analysis.

[3] Robust standard errors were obtained via a modification of the summary() function written by the author of the Economic Theory Blog specifically with the purpose of replicating the STATA analysis 
method which von Hippel and Huron (2020) used in their original study. This modification was retrieved from https://raw.githubusercontent.com/IsidoreBeautrelet/economictheoryblog/master/robust summary.R.

[4] Although extensive discussions of Butler's (1989) Intervallic Rivalry Theory and related concepts of tonality based on sequential real-time processing rather than on retrospective distributional aspects are absent from von Hippel and Huron's (2020) account, the second author did acknowledge in a footnote in his Sweet Anticipation (Huron, 2006) that "it would appear that this research seems to give short shrift to the Browne/Butler/Brown approach to tonality. However, as we have demonstrated, order matters in the organization of tone rows, which was precisely the main point of contention between Krumhansl and Butler" (p. 406).

\section{REFERENCES}

Agres, K., Abdallah, S., \& Pearce, M. (2018). Information-theoretic properties of auditory sequences dynamically influence expectation and memory. Cognitive Science, 42(1), 43-76. https://doi.org/10.1111/cogs.12477

Ball, P. (2011). Schoenberg, serialism and cognition: whose fault if no one listens? Interdisciplinary Science Reviews, 36(1), 24-41. https://doi.org/10.1179/030801811X12941390545645

Barlow, H., \& Morgenstern, S. (1983). A dictionary of musical themes. London, UK: Faber \& Faber.

Brown, J. L. (2016). The psychological representation of musical intervals in a twelve-tone context. Music Perception, 33(3), 274-286. https://doi.org/10.1525/mp.2016.33.3.274

Butler, D. (1989). Describing the perception of tonality in music: a critique of the tonal hierarchy theory and a proposal for a theory of intervallic rivalry. Music Perception, 6(3), 219-241. https://doi.org/10.2307/40285588

Christiansen, M. H., \& Chater, N. (2016). The now-or-never bottleneck: a fundamental constraint on language. Behavioral and Brain Sciences, 39. https://doi.org/10.1017/S0140525X1500031X

Cosmides, L., \& Tooby, J. (2000). Consider the source: the evolution of adaptations for decoupling and metarepresentation. In D. Sperber (Ed.), Metarepresentations: a multidisciplinary perspective (pp. 53115). Oxford, UK: Oxford University Press.

Dibben, N. (1994). The cognitive reality of hierarchic structure in tonal and atonal music. Music Perception, 12(1), 1-25. https://doi.org/10.2307/40285753

Dienes, Z., \& Longuet-Higgins, C. (2004). Can musical transformations be implicitly learned? Cognitive Science, 28, 531-558. https://doi.org/10.1207/s15516709cog2804_2

Hansen, N. C., \& Pearce, M. T. (2014). Predictive uncertainty in auditory sequence processing. Frontiers in Psychology, 5, 1052. https://doi.org/10.3389/fpsyg.2014.01052

Hansen, N. C., Vuust, P., \& Pearce, M. (2016). "If you have to ask, you'll never know:" effects of specialised stylistic expertise on predictive processing of music. PLOS ONE, 11(10), e0163584. https://doi.org/10.1371/journal.pone.0163584

Hansen, N. C., Kragness, H., Vuust, P., Trainor, L., \& Pearce, M. (under review). Predictive uncertainty underlies auditory boundary perception.

Hanson, R. (1983). Webern's chromatic organisation. Music Analysis, 2(2), 135-149. https://doi.org/10.2307/854246

Hauer, J. M. (1920). Vom Wesen des Musikalischen. Vienna, Austria: Waldheim-Eberle. 
Headlam, D. J. (1996). The music of Alban Berg. New Haven, CT: Yale University Press.

von Hippel, P. T., \& Huron, D. (2020). Tonal and "anti-tonal" cognitive structure in Viennese twelve-tone rows. Empirical Musicology Review, 15(1-2), 108-118. https://doi.org/10.18061/emr.v15i1-2.7655

Huron, D. B. (2006). Sweet anticipation: music and the psychology of expectation. Cambridge, MA: MIT Press. https://doi.org/10.7551/mitpress/6575.001.0001

Huron, D., \& von Hippel, P. (2000). Tonal and contra-tonal structure of Viennese twelve-tone rows. Paper presented at the Society for Music Theory Conference, Toronto, Canada.

Jarman, D. (1985). The music of Alban Berg. Berkeley/Los Angeles, CA: University of California Press.

Koenker, R. (2020). quantreg: quantile regression. $R$ package version 5.55 . Retrieved from https://CRAN.R-project.org/package=quantreg

Krumhansl, C. L., Sandell, G. J., \& Sergeant, D. C. (1987). The perception of tone hierarchies and mirror forms in twelve-tone serial music. Music Perception, 5(1), 31-77. https://doi.org/10.2307/40285385

Krumhansl, C. L. (1990). Cognitive foundations of musical pitch. New York, NY: Oxford University Press.

Krumhansl, C. L., \& Kessler, E. J. (1982). Tracing the dynamic changes in perceived tonal organization in a spatial representation of musical keys. Psychological Review, 89, 334-368. https://doi.org/10.1037/0033-295X.89.4.334

Krumhansl, C. L., \& Schmuckler, M. A. (1986). Key-finding in music: an algorithm based on pattern matching to tonal hierarchies. Paper presented at the 19th Annual Meeting of the Society of Mathematical Psychology, Cambridge, MA.

de Lannoy, C. (1972). Detection and discrimination of dodecaphonic series. Journal of New Music Research, 1(1), 13-27. https://doi.org/10.1080/09298217208570157

Lewandowsky, S., \& Murdock Jr, B. B. (1989). Memory for serial order. Psychological Review, 96(1), $25-$ 57. https://doi.org/10.1037/0033-295X.96.1.25

Lerdahl, F. (1992). Cognitive constraints on compositional systems. Contemporary Music Review, 6(2), 97-121. https://doi.org/10.1080/07494469200640161

Lerdahl, F. (2001). Tonal pitch space. New York, NY: Oxford University Press.

London, J., von Hippel, P. T., Huron, D., Cartano, J., Kingery, K., Olsen, B., \& Santelli, T. (2002, June 24). Row forms in the serial works of Schoenberg, Berg, and Webern. Stanford, CA: Center for Computer Assisted Research in the Humanities. Retrieved from: http://www.ccarh.org/publications/data/humdrum/ tonerow/

Margulis, E. H. (2014). On repeat: how music plays the mind. New York, NY: Oxford University Press. https://doi.org/10.1093/acprof:oso/9780199990825.001.0001

Maylor, E. A. (2002). Serial position effects in semantic memory: reconstructing the order of verses of hymns. Psychonomic Bulletin \& Review, 9(4), 816-820. https://doi.org/10.3758/BF03196340

Morrison, S. J., Demorest, S. M., \& Pearce, M. T. (2018). Cultural distance: a computational approach to exploring cultural influences on music cognition. In M. H. Thaut \& D. A. Hodges (Eds), The Oxford Handbook of Music and the Brain (pp. 41-65). Oxford, UK: Oxford University Press.

https://doi.org/10.1093/oxfordhb/9780198804123.013.3 
Ockelford, A., \& Sergeant, D. (2013). Musical expectancy in atonal contexts: musicians' perception of "antistructure." Psychology of Music, 41(2), 139-174. https://doi.org/10.1177/0305735612442582

Pearce, M. T. (2005). The construction and evaluation of statistical models of melodic structure in music perception and composition (Doctoral dissertation, City University London).

Pearce, M. T., Müllensiefen, D., \& Wiggins, G. A. (2010). The role of expectation and probabilistic learning in auditory boundary perception: a model comparison. Perception, 39(10), 1367-1391. https://doi.org/10.1068/p6507

Pedersen, P. (1975). The perception of octave equivalence in twelve-tone rows. Psychology of Music, 3(2), 3-8. https://doi.org/10.1177/030573567532001

R Core Team (2018). R: a language and environment for statistical computing. R Foundation for Statistical Computing, Vienna, Austria. Retrieved from: https://www.R-project.org/

Schaffrath, H. (1992). The ESAC databases and MAPPET software. Computing in Musicology, 8, 66.

Schaffrath, H. (1993). The ESAC electronic songbooks. Computing in Musicology, 9, 78.

Schoenberg, A. (1941/1950). Composition with twelve tones. In D. Newlin (Ed.), Style and Idea (pp. 102143). New York, NY: Philosophical Library.

Schmuckler, M. (2016). Tonality and contour in melodic processing. In S. Hallam, I. Cross, \& M. Thaut (Eds), The Oxford Handbook of Music Psychology (pp. 143-165). New York, NY: Oxford University Press. https://doi.org/10.1093/oxfordhb/9780198722946.013.14

Sears, D. R., Pearce, M. T., Caplin, W. E., \& McAdams, S. (2018). Simulating melodic and harmonic expectations for tonal cadences using probabilistic models. Journal of New Music Research, 47(1), 29-52. https://doi.org/10.1080/09298215.2017.1367010

Sears, D. R., Pearce, M. T., Spitzer, J., Caplin, W. E., \& McAdams, S. (2019). Expectations for tonal cadences: sensory and cognitive priming effects. Quarterly Journal of Experimental Psychology, 72(6), 1422-1438. https://doi.org/10.1177/1747021818814472

Taruskin, R. (2004). The poietic fallacy. The Musical Times, 145(1886), 7-34. https://doi.org/10.2307/4149092 